\title{
Spectral distribution of photoelectric quantum yield of thin-film Au-CdTe diode structure
}

\author{
E.V. Grushko ${ }^{1}$, O.L. Maslyanchuk ${ }^{1}$, X. Mathew ${ }^{2}$, V.V. Motushchuk ${ }^{1}$, L.A. Kosyachenko ${ }^{1}$, E.A. Streltsov ${ }^{3}$ \\ ${ }^{1}$ Chernivtsi National University, 58012 Chernivtsi, Ukraine \\ ${ }^{2}$ Centro de Investigacion en Energia-UNAM, 62580, Temixco, Morelos, Mexico \\ ${ }^{3}$ Belarussian State University, 4, F. Skaryna Ave., Minsk 220050, Belarus \\ E-mail: lakos@chv.ukrpack.net
}

\begin{abstract}
A study of the $\mathrm{Au} / \mathrm{CdTe}$ Schottky diodes fabricated by vacuum evaporation of a semitransparent $\mathrm{Au}$ layer over an electrodeposited CdTe thin film is reported. The theoretical model of the photocurrent spectra for an $\mathrm{Au} / \mathrm{CdTe}$ Schottky diode based on the continuity equation and incorporating the surface recombination losses does not explain the measured spectra in the entire range of wavelengths, particularly the abovementioned decay in the short-wavelength region. The satisfactory description of the measured spectra is achieved by proposing a model, in which the surface recombination along with the Schottky effect resulted in the presence of a dead layer in the space-charge region is taken into account. By varying the parameters such as uncompensated carrier concentration and carrier lifetime, the above model can explain the actual photoresponse spectra.
\end{abstract}

Keywords: CdTe thin-film, CdTe Schottky diode, surface recombination, spectral response.

Manuscript received 23.11.07; accepted for publication 19.12.07; published online 31.01.08.

\section{Introduction}

Thin-film CdTe-based photovoltaic devices on a metal foil are considered prospective for the large-scale production of photosensitive devices $[1,2]$. CdTe is a direct bandgap semiconductor and exhibits high optical absorption in the $h v \geq E_{\mathrm{g}}$ photon-energy region. Therefore, the high quantum efficiency is expected for $\mathrm{CdTe}$ photovoltaic devices in a wide range of semiconductor intrinsic absorption. However, the spectral dependence of the photoelectric quantum efficiency of $\mathrm{Au} / \mathrm{CdTe}$ thin-film solar cells on metal foils exhibits considerable non-uniformity [3]. Furthermore, a considerable decay in the quantum efficiency can be observed for wavelengths shorter than $\sim 450 \mathrm{~nm}$ [4] which is the region of a noticeable portion of solar radiation. In this paper, we develop a theoretical model based on the continuity equation for the photoelectric quantum yield which is applied to explain the spectral response data of $\mathrm{Au} / \mathrm{n}-\mathrm{CdTe}$ thinfilm Schottky diodes. It is shown that the observed features in the spectra can be explained by taking the Schottky effect into account.

\section{Experimental}

CdTe layers $\sim 1 \mu \mathrm{m}$ thick were prepared on the stainless steel substrates ( $50 \mu \mathrm{m}$ thick) by electrodeposition [2]. The Schottky devices were formed by vacuum evaporation of a semitransparent (10-12 nm thick) layer of $\mathrm{Au}$ over the $\mathrm{CdTe}$ surface at a substrate temperature of $150{ }^{\circ} \mathrm{C}$. The spectral characteristics of the samples in the range of $300-900 \mathrm{~nm}$ were recorded by using a photoresponse system equipped with a quartz halogen lamp. The relative distribution of the photon flux (rather than the radiation energy) at the outlet slit of the system was determined using a calibrated Si photodiode.

Figure 1 illustrates the spectral response of three samples reflecting the most common features of the photoelectric quantum efficiency of $\mathrm{Au} / \mathrm{CdTe}$ diodes. In a number of cases, a fairly uniform sensitivity in the range of $500-700 \mathrm{~nm}$ with a steep decay at shorter wavelengths was observed (sample N3). For other samples, an increase in the spectral response in the 400-500-nm regions followed by the decay and a shoulder at $\lambda \approx 350 \mathrm{~nm}$ was also observed (samples N1 and N2). The shape of the spectral curve can be 
somewhat modified by varying the temperature, but the common features of the spectra remain unchanged (Fig. 2).

\section{Theoretical treatment}

The continuity equation for minority carriers (holes in this case) has the form

$D_{\mathrm{p}} \frac{d^{2} \Delta p}{d x^{2}}-\frac{\Delta p}{\tau_{p}}+\Phi_{0} \alpha \exp (-\alpha x)=0$,

where $\Delta p$ is the excess concentration of holes, $\tau_{p}$ is the hole lifetime, $\alpha$ is the absorption coefficient, and $\Phi_{0}$ is the number of photons incident per second on a unit area of the diode (corrected for reflection losses).

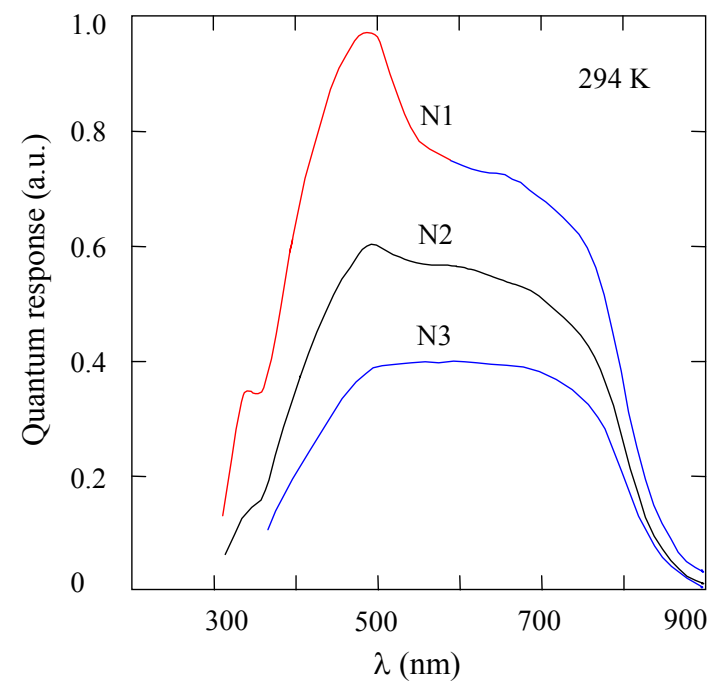

Fig. 1. Representative room-temperature spectral response obtained from $\mathrm{Au} / \mathrm{n}$-CdTe thin-film photovoltaic devices on metal substrates.

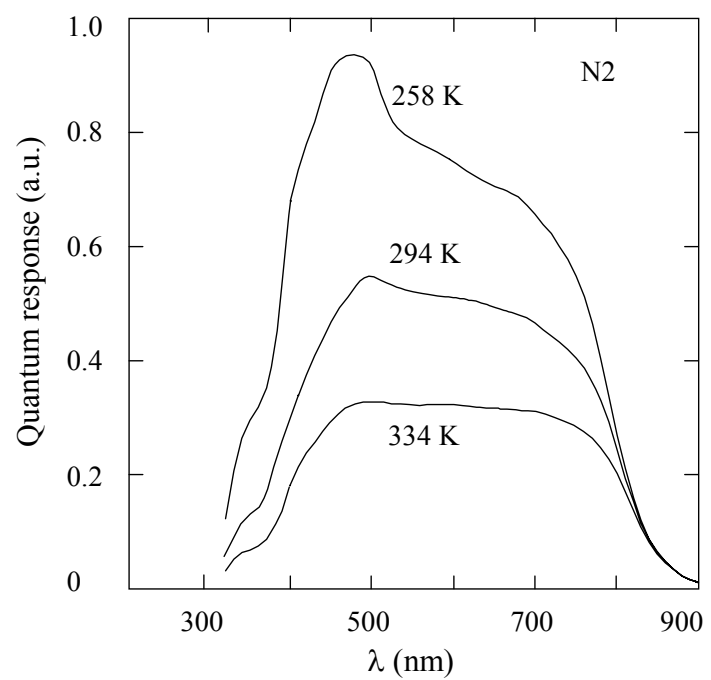

Fig. 2. Spectral response of the $A u / n-C d T e ~ d e v i c e ~(N 2)$ at different temperatures.
Equation (1) is solved using the boundary conditions. The $\Delta p$ values for the depletion layer and the neutral part of the film should coincide at their interface $(x=W)$, whereas the surface recombination at the front surface of the film $(x=0)$ should be compensated by the diffusion inflow of holes, i.e.,

$S_{p} \Delta p(0)=-\left.D_{p} \frac{\Delta p(W)}{d x}\right|_{x=0}$.

The exact solution of the continuity equation with regard to the surface recombination at the metal/semiconductor interface leads to a rather cumbersome and non-visual expression for the photoelectric quantum efficiency $\eta(\alpha)[5]$ :

$$
\begin{aligned}
& \eta(\alpha)=\frac{1+\frac{S}{D_{n}} \exp \left(-\frac{W^{2}}{W_{0}^{2}}\right)\left[A(\alpha)-D_{1}(\alpha)\right]}{1+\frac{S}{D_{n}} \exp \left(-\frac{W^{2}}{W_{0}^{2}}\right) B}- \\
& -\frac{\exp (-\alpha W)}{1+\alpha L_{p}}-D_{2}(\alpha),
\end{aligned}
$$

where $S$ is the surface recombination velocity, $D_{n}$ is the electron diffusion coefficient, $L_{p}$ is the hole diffusion length, and $W$ is the depletion layer width,

$W=\sqrt{\frac{2 \varepsilon \varepsilon_{0}\left(\varphi_{0}-q V\right)}{q^{2}\left(N_{d}-N_{a}\right)}}$,

$\varepsilon_{0}$ and $\varepsilon$ are, respectively, the free space and semiconductor permittivities, $\varphi_{0}$ is the barrier height at the semiconductor side $\left(\varphi_{0}=q V_{b i}\right.$, where $V_{b i}$ is the built-in potential), $q$ is the electron charge, $V$ is the applied voltage, and $N_{d}-N_{a}$ is the uncompensated donor concentration. In Eq. (3), the following designations are also used:

$$
\begin{aligned}
& W_{0}=\sqrt{\frac{2 \varepsilon \varepsilon_{0} k T}{q^{2}\left(N_{d}-N_{a}\right)}}, \\
& A(\alpha)=\int_{0}^{W} \exp \left[-\alpha x+\left(\frac{x-W}{W_{0}}\right)^{2}\right] d x, \\
& B=\int_{0}^{W} \exp \left(\frac{x-W}{W_{0}}\right)^{2} d x
\end{aligned}
$$

( $W_{0}=\sqrt{2} W_{\mathrm{D}}$, where $W_{\mathrm{D}}$ is the effective Debye length). Equation (3) can be essentially simplified. At the boundary between the depletion and neutral regions $(x=W)$, the photogenerated holes are entrained by the strong electric field and, hence, one may put $\Delta p(W)=0$. This means that, introducing an inessential error, the terms $D_{1}(\alpha)$ and $D_{2}(\alpha)$ in Eq. (3) can be neglected [5]. The 
integrand in Eq. (6) is a decreasing exponential function $f(x)$. Therefore, the integration in Eq. (6) can be substituted by the product of the maximum value of the integrand $f_{\max }(0)=\exp \left(W / W_{0}\right)^{2}$ by its "half-width". The latter is determined by the point, at which the value of the function $f(x)$ is smaller than its maximum value by a factor of $e=2.71$. Thus, the value $x$ such that the above condition is fulfilled can be derived from the equation

$$
\exp \left[-\alpha x+\left(\frac{x-W}{W_{0}}\right)^{2}\right]=\exp \left(\frac{W}{W_{0}}\right)^{2} e^{-1}
$$

which reduces to the quadratic equation

$$
x^{2}-\left(\alpha W_{0}^{2}+2 W\right) x+W_{0}^{2}=0 \text {. }
$$

A solution of Eq. (9) is

$$
\begin{aligned}
& x=\frac{\alpha W_{0}^{2}+2 W}{2}\left(1-\sqrt{1-\frac{4 W_{0}^{2}}{\left(\alpha W_{0}^{2}+2 W\right)^{2}}}\right) \approx \\
& \approx\left(\alpha+\frac{2 W}{W_{0}^{2}}\right)^{-1}=\left(\alpha+\frac{2}{W} \frac{\varphi_{0}-q V}{k T}\right)^{-1}
\end{aligned}
$$

(here, the expansion in a series $\sqrt{1-x} \approx 1-x / 2$ has been used taking into consideration that the second term of the radicand is much less than 1). Similarly, Eq. (7) can be represented in the form

$$
x \approx\left(\frac{2}{W} \frac{\varphi_{0}-q V}{k T}\right)^{-1} .
$$

Thus, instead of Eq. (3), the photoelectric quantum efficiency can be written as

$$
\eta(\alpha)=\frac{1+\frac{S}{D_{n}}\left(\alpha+\frac{2}{W} \frac{\varphi_{0}-q V}{k T}\right)^{-1}}{1+\frac{S}{D_{n}}\left(\frac{2}{W} \frac{\varphi_{0}-q V}{k T}\right)^{-1}}-\frac{\exp (-\alpha W)}{1+\alpha L_{p}} .
$$

It is seen from Eq. (12) that the effect of surface recombination is enhanced when either $\alpha$ or $W$ increases. An increase in $\alpha$ indicates the stronger absorption near the surface, and an increase in $W$ means that, as the depletion region widens, the electric field becomes weak). Of course, in the absence of surface recombination $(S=0)$, Eq. (12) reduces to the well-known Gartner formula [6]:

$\eta(\alpha)=1-\frac{\exp (-\alpha x)}{1+\alpha L_{p}}$.

A comparison of the calculated values of $\eta(\alpha)$ using both Eqs. (3) and (12) in a wide range of $\alpha$ values is shown in Fig. 3. In computing $\eta$, the barrier height $\varphi_{0}$ was taken $1 \mathrm{eV}$ and the electron diffusion coefficient $D_{n}=k T \mu_{n} / q=25 \mathrm{~cm}^{2} / \mathrm{s}$ for the electron mobility $\mu_{n}=$
$1000 \mathrm{~cm}^{2} /(\mathrm{V} \cdot \mathrm{s})$. As is known, even the purest CdTe contains residual impurities of the order of $10^{15}$. $10^{17} \mathrm{~cm}^{-3}[7,8]$. Therefore, the observed resistivity of $10^{6}-10^{7} \mathrm{Ohm} \cdot \mathrm{cm}$ for electrodeposited films reveals a partially compensated conduction in the material. The actual compensation is far away from a total compensation, because the measured resistivity of the CdTe film is lower than the intrinsic conductivity which is about $10^{10} \mathrm{Ohm} \cdot \mathrm{cm}\left(E_{\mathrm{g}}=1.46 \mathrm{eV}, m_{n}=0.11 m_{0}, m_{p}=\right.$ $\left.0.35 m_{0}, T=300 \mathrm{~K}\right)$. Considering the above point, the uncompensated donor concentration $N_{d}-N_{a}$ was taken to be the average value of $10^{16} \mathrm{~cm}^{-3}$, which corresponds to $W \approx 0.3 \mu \mathrm{m}(\varepsilon=10.6$ for $\mathrm{CdTe})$. Due to a high concentration of deep levels in the bandgap, the carrier lifetime in CdTe is rather short, and the hole lifetime was taken in the calculation as $10^{-8} \mathrm{~s}$; correspondingly, the hole diffusion length $L_{p}=\left(\tau D_{p}\right)^{1 / 2} \approx 1 \mu \mathrm{m}$. The surface recombination velocity $S$ was taken to be its maximum value, which is equal, in turn, to the electron velocity in a semiconductor at the interface. This velocity is equal to $A^{*} T^{2} / q N_{c}$, where $A^{*}=$ $q m_{n} k^{2} / 2 \pi^{2} h^{3}$ is the effective Richardson constant [9]. Taking $m_{n}=0.11 \mathrm{~m}_{0}$, the electron velocity $V_{n} \approx 10^{7} \mathrm{~cm} / \mathrm{s}$.

As can be seen from Fig. 3, the curves computed by Eqs. (3) and (12) are close to each other over the entire range of the absorption coefficient. Note that the difference remains within few percents even if one varies other parameters $\left(\tau, S, N_{d}-N_{a}\right)$ in reasonable ranges. Thus, it is clear that Eq. (12) works very well as Eq. (3).

In order to plot the $\eta(h v)$ dependences, a knowledge of the absorption curve $\alpha(h v)$ for CdTe is required. The evaluation of the absorption coefficient in the higher energy region of the spectrum is not a simple task, since it requires either very thin samples or a combination of optical techniques (transmittance, reflectance, etc.). We have estimated the values of $\alpha(h v)$ in the absorption edge region from the transmittance spectra. For the region of higher photon energies $(h v=1.5-5.4 \mathrm{eV})$, the $\alpha(h v)$ values were taken from [10] (Fig. 4).

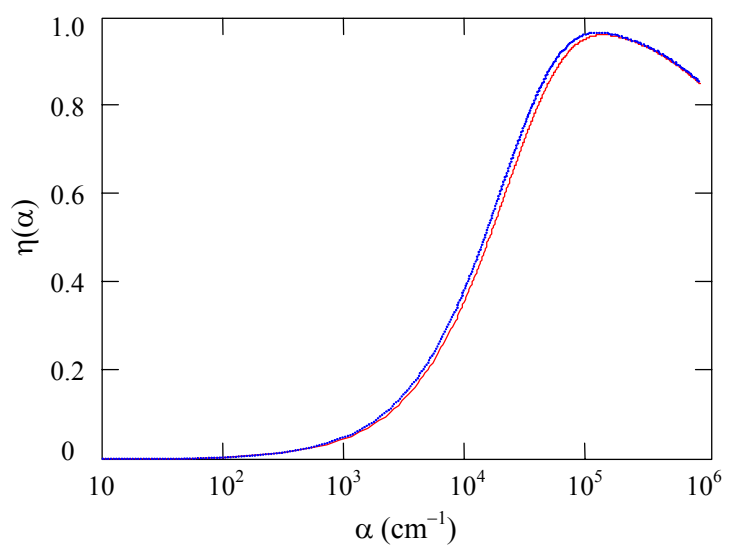

Fig. 3. Dependence of the photoelectric quantum efficiency $\eta$ on the absorption coefficient $\alpha$ computed by Eq. (3) (solid line) and by Eq. (12) (dashed line). 


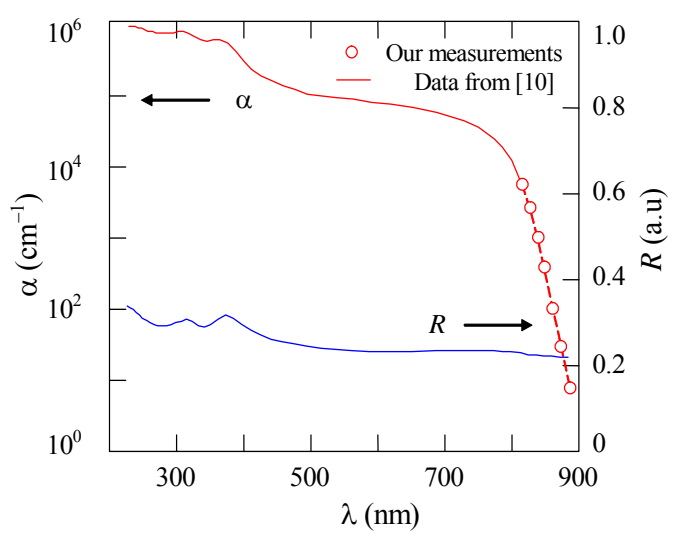

Fig. 4. Spectral dependences of the absorption coefficient and the reflection coefficient used in this work.

Figure 5 shows two groups of photoresponse spectra generated using Eq. (12) for different hole lifetimes $\tau_{p}$ and two different values of the surface recombination velocity $S=10^{7} \mathrm{~cm} / \mathrm{s}$ and $S=0$. The magnitudes $\tau_{p}=10^{-6} \mathrm{~s}$ and $\tau_{p}=10^{-12} \mathrm{~s}$ correspond to the maximum achievable values of the hole lifetimes in CdTe single crystals and the reported values for electrodeposited CdTe films, respectively. The uncompensated carrier concentration was taken to $3 \times 10^{15} \mathrm{~cm}^{-3}$, which is close to the minimum possible value (in accordance with the mentioned above).

It is clear from Fig. 5 that the effect of surface recombination turns out rather weak for the actual parameters $\tau_{p}, S$, and $N_{d}-N_{a}$. Indeed, in the shortwavelength region (where surface recombination is most effective), the decay of the response does not exceed 10$15 \%$ as compared with its maximum. This indicates that a strong electric field exists in the depletion region. For $\varphi_{0}=1 \mathrm{eV}, V=0$, and $N_{d}-N_{a}=10^{15} \mathrm{~cm}^{-3}$ (as an example of a thick depletion layer), the electric field strength at the interface $F=2\left(\varphi_{0}-e V\right) / q W$ is equal to $\sim 2 \times 10^{4} \mathrm{~V} / \mathrm{cm}$, and, for higher $N_{d}-N_{a}$ values, the electric field becomes stronger. Note that a shoulder-like hump at $\lambda \approx 350 \mathrm{~nm}$ is due to the features observed in the absorption and reflection spectra shown in Fig. 4.

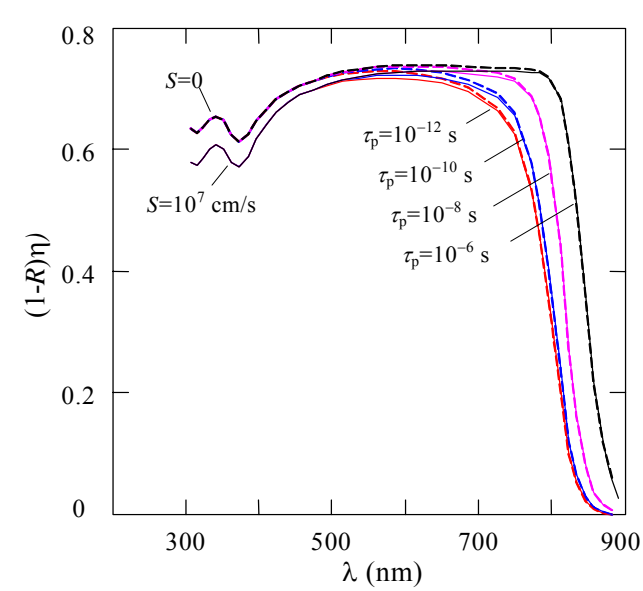

Fig. 5. Spectral response of $\mathrm{Au} / \mathrm{n}-\mathrm{CdTe}$ computed for different hole lifetimes $\tau_{p}$, with the surface recombination velocities $S=$ $10^{7} \mathrm{~cm} / \mathrm{s}$ and $S=0 ; N_{d}-N_{a}=3 \times 10^{15} \mathrm{~cm}^{-3}$.
Figure 6 is the computed spectral response illustrating the effect of uncompensated donor impurities in an $\mathrm{Au} / \mathrm{n}$-CdTe diode. In this calculation, the values of $S$ and $\tau_{p}$ are taken as $10^{7} \mathrm{~cm} / \mathrm{s}$ and $10^{-10} \mathrm{~s}$, respectively. It can be seen from Fig. 6 that, as the uncompensated donor concentration varies, the shape of the $\eta(\lambda)$ curves undergo significant changes. As $N_{d}-N_{a}$ decreases, the spectral response in the $\lambda>400 \mathrm{~nm}$ region enhances noticeably, which is due to the expansion of the depletion layer and, hence, more efficient collection of photogenerated carriers from the bulk of the film. On the other hand, as the depletion layer widens, the electric field becomes weaker which is favorable for the surface recombination. This effect is clearly demonstrated by the graph, for which $N_{d}-N_{a}$ is $10^{14} \mathrm{~cm}^{-3}$ (dashed line in Fig. 6): the surface recombination causes a significant decay in the response in the direction of decreasing the wavelength. But in practical cases, the $N_{d}-N_{a}$ concentration in CdTe-based thin-film photovoltaic devices is in the range $10^{15}-10^{17} \mathrm{~cm}^{-3}$ (the range of the residual impurity and defect concentrations), i.e., the value $N_{d}-N_{a}=10^{14} \mathrm{~cm}^{-3}$ is unreal [7, 8].

A comparison of the computed spectra in Figs. 5 and 6 with the experimental results presented in Figs. 1 and 2 shows that, by varying the parameters, one can model the spectral response of $\mathrm{Au} / \mathrm{CdTe}$ Schottky diodes and obtain spectra similar to the experimental curves but only in the wavelength region $\lambda>400 \mathrm{~nm}$. In the region of shorter wavelengths, we were not successful in generating a model to fit the experimental data.

It should be noted that the discussed model does not account for the fact that, in thin-film CdTe solar cells, the thickness of the absorber layer does not exceed a few microns (in the above-reported samples, it is even thinner). In this case, in the spectral region where the absorption coefficient is relatively small $\left(<10^{4} \mathrm{~cm}^{-1}\right)$, recombination can occur at the back surface. This is not taken into account in Eqs. (3) and (12), since the excess hole concentration is set equal to 0 at $x=\infty[5]$.

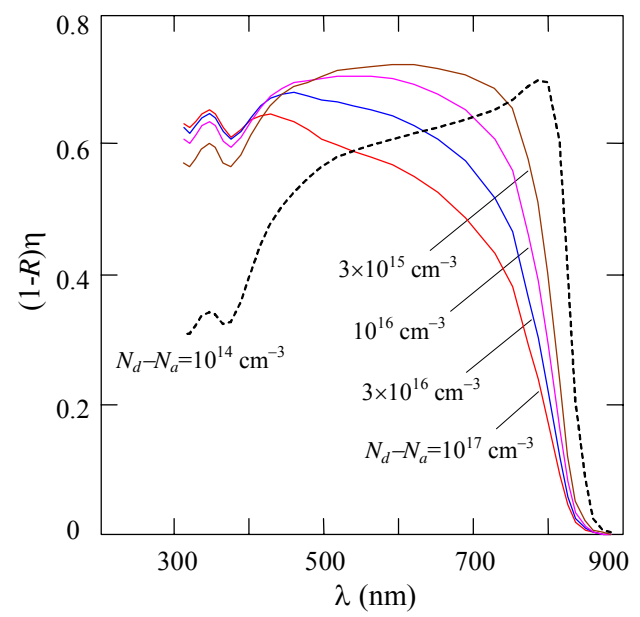

Fig. 6. Spectral response computed for different uncompensated donor concentrations $N_{d}-N_{a}\left(\tau_{p}=10^{-10} \mathrm{~s}\right.$, $S=10^{7} \mathrm{~cm} / \mathrm{s}$ ). 
However, (i) this effect is weak for moderately thick films and (ii) it manifests itself in the long-wavelength region rather than in the short-wavelength region.

\section{Taking into account the Schottky effect}

The Schottky effect lies not only in the lowering of the energy barrier at the metal/semiconductor interface but also in developing a layer at the semiconductor surface, where the electric field acts in a direction opposite to that in the rest of the space-charge region (the field attracts electrons to the semiconductor surface) (Fig. 7). We will name this layer as "dead" in the sense that electron-hole pairs photogenerated in the layer do not contribute to the photocurrent formation. The thickness of this layer depends on the electric field strength and, hence, on the uncompensated donor concentration. Taking the attractive image force into account, the electron potential energy as a function of the distance from the semiconductor surface is given by the relation [9]

$\varphi(x)=\frac{q^{2}}{16 \pi \varepsilon \varepsilon_{0} x}+q F(x) x$,

where $F(x)$ is the electric field strength in the depletion region,

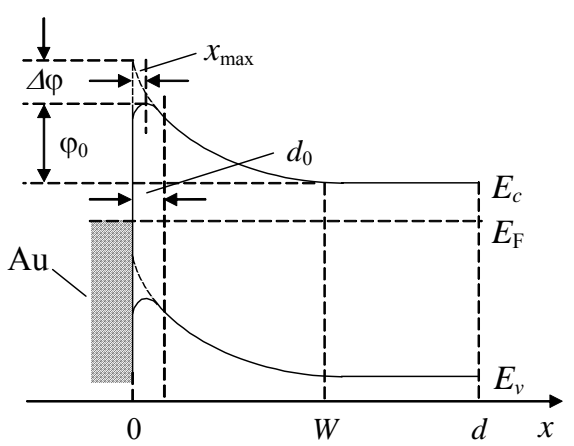

(a)

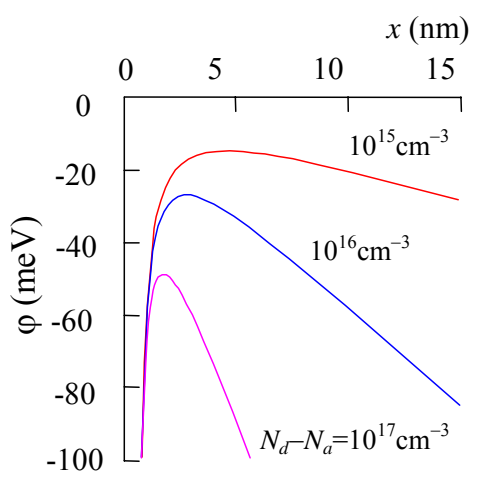

(b)

Fig. 7. Energy-band diagram of an $\mathrm{Au} / \mathrm{n}-\mathrm{CdTe}$ contact incorporating the Schottky effect (a). The shape of the electron potential energy near the metal/CdTe interface calculated for different concentrations of uncompensated donors (b).
$F(x)=\frac{2\left(\varphi_{0}-e V\right)}{e W}\left(1-\frac{x}{W}\right)$.

Figure $7 \mathrm{~b}$ represents the curves calculated by Eq. (14) with the use of Eqs. (15) and (4) for three $N_{d}-N_{a}$ values. When $N_{d}-N_{a}$ decreases, the barrier lowers, and the maximum on the $\varphi(x)$ curve concurrently moves away from the semiconductor surface. It should be borne in mind that the "dead" layer thickness is greater than $x_{\max }$ (Fig. 7a), since the electric field strength $F=-(1 / q) d \varphi / d x$ is zero at $x=x_{\max }$ and gradually increases with distance from $x_{\max }$. Hence, to the right of the maximum, the electric field is weak for some distance. We denote the effective thickness of the dead layer by $d_{0}$. As follows from the above discussion, its action on the photoresponse of a diode manifests itself in three ways: (i) the carriers photogenerated in this layer do not contribute to the photocurrent, (ii) the absorption in the dead layer reduces the amount of radiation reaching the active depletion layer, and (iii) the dead layer is favorable for the recombination losses at the interface.

Taking the Schottky effect into account, Eq. (12) takes the form:

$$
\begin{aligned}
& \eta(\alpha)=\exp \left(-\alpha d_{0}\right) \times \\
& \times\left[\frac{1+\frac{S}{D_{n}}\left(\alpha+\frac{2}{W} \frac{\varphi_{0}-q V}{k T}\right)^{-1}}{1+\frac{S}{D_{n}}\left(\frac{2}{W} \frac{\varphi_{0}-q V}{k T}\right)^{-1}}-\frac{\exp (-\alpha W)}{1+\alpha L_{p}}\right]
\end{aligned}
$$

(we ignore the barrier lowering $\Delta \varphi$ and the barrier narrowing due to the dead layer presence, since $\Delta \varphi<<\varphi_{0}$ and $d_{0}<<W$ ).

The quantum response spectra calculated by using Eq. (16) for different dead layer thicknesses $d_{0}$ and two values of $N_{d}-N_{a}$ are presented in Fig. $8 \mathrm{a}$ and b.

As can be seen in Fig. 8, the incorporation of the Schottky effect gives an explanation for the steep decay of the photoresponse in the short-wavelength region $(\lambda<400 \mathrm{~nm})$. Note that the $\eta(\alpha)$ dependences computed by using Eq. (16) are similar to the experimental curves shown in Figs. 1 and 2. As already mentioned above, the significant reduction of the response in the region $\lambda>450 \mathrm{~nm}$ for the spectra shown by dashed lines in Fig. $8 \mathrm{a}$ is due to a narrow depletion layer (a higher concentration of uncompensated donors). As a result, a considerable portion of the long-wavelength radiation is absorbed outside the depletion layer and, hence, they are not efficiently collected, since the hole diffusion length is small $\left(\sim 0.01 \mu \mathrm{m}\right.$ for $\left.\tau_{p}=10^{-9} \mathrm{~s}, D_{p}=2 \mathrm{~cm}^{2} /(\mathrm{V} \cdot \mathrm{s})\right)$. In the region $\lambda<450 \mathrm{~nm}$, the absorption coefficient is more than $10^{5} \mathrm{~cm}^{-1}$ (Fig. 4), and the majority of photons are absorbed in the depletion layer even for thin depletion layers. It should be emphasized that the hump observed for samples N1 and N2 at about $475 \mathrm{~nm}$ (Fig. 1, $N_{d}-N_{a}$ $=10^{17} \mathrm{~cm}^{-3}$ ) is the combined effect: (i) in the intermediate wavelength region, the portion of the long- 
wavelength radiation is absorbed in the neutral region (the depletion layer is narrow), and the photoresponse enhances as the wavelength shortens, (ii) in the shortwavelength region, the Schottky effect causes the photoresponse to decrease due to the presence of a dead layer. If the depletion layer is sufficiently wide, nearly all of the photons are absorbed in the depletion layer, where the charge collection is effective, and we have a relatively uniform response in the 400-800-nm wavelength range. At the shortest wavelengths, the photoresponse decreases again due to the presence of a dead layer. As a result, the photoresponse spectrum is similar to that of sample N3 in Fig. 1.

It follows from Fig. 8 that, for $\tau_{p}=10^{-9} \mathrm{~s}$, (a) the quantum efficiency is greater than that for $\tau_{p}=10^{-12} \mathrm{~s}$ (b), which is particularly noticeable when the carrier concentration is high $\left(N_{d}-N_{a}=10^{17} \mathrm{~cm}^{-3}\right)$. The reason is that, for $\tau_{p}=10^{-9} \mathrm{~s}$, the hole diffusion length becomes as long as $\sim 0.4 \mu \mathrm{m}$ and a remarkable part of photocarriers generated outside the depletion region are collected.

(a)

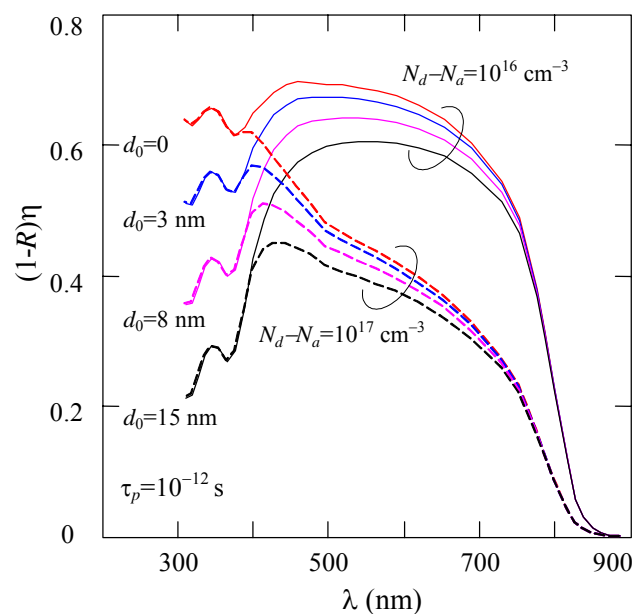

(b)

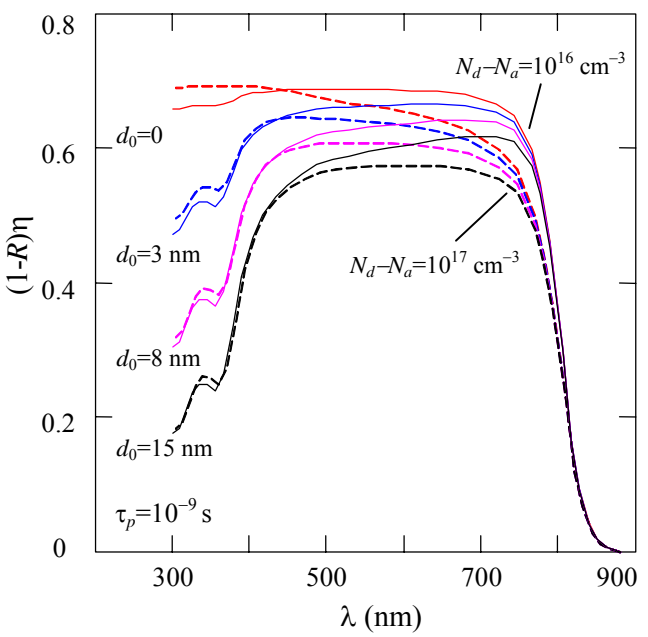

Fig. 8. Spectral response computed for different thicknesses of the dead layer $d_{0}$ and different $N_{d}-N_{a}$ values: (a) hole lifetime $\tau_{p}=10^{-12} \mathrm{~s}$ and (b) $\tau_{p}=10^{-9} \mathrm{~s}\left(S=10^{7} \mathrm{~cm} / \mathrm{s}, R=0.3\right)$.
In this paper, we have made an attempt to compare semiqualitatively the measured photocurrent spectra with our theoretical models. A quantitative comparison is unjustified since the reflectance spectrum of a diode with $\mathrm{Au}$ coating is unknown, and also the absorption characteristic of polycrystalline $\mathrm{CdTe}$ can differ considerably from that of the single crystal.

\section{Conclusions}

The photoresponse spectra of the Au/CdTe Schottky diodes developed on metal foil substrates have been measured. The theoretical model of the photocurrent spectra for an $\mathrm{Au} / \mathrm{CdTe}$ Schottky diode based on the continuity equation and incorporating the surface recombination losses does not explain the measured spectra in the entire range of wavelengths, particularly the large photoresponse decay in the $\lambda<450 \mathrm{~nm}$ region. The satisfactory description of the measured spectra is achieved by proposing a model, in which the surface recombination along with the Schottky effect is taken into account. By varying the parameters such as uncompensated carrier concentration and carrier lifetime, the above model can explain the actual photoresponse spectra.

\section{Acknowledgements}

The CdTe films used in this work were prepared as a part of the projects funded by PAPIIT-UNAM (IN115102) and CONACYT (38542-U). The study was supported by the State Foundation for Fundamental Investigations (Ministry of Education and Technology, Ukraine) within the Agreement F14/259-2007.

\section{References}

1. J.C. McClure, V.P. Singh, G.B. Lush, E. Clarc, G. Thompson // Solar Energy Materials \& Solar Cells 55, p. 141 (1998).

2. X. Mathew, G.W. Thompson, V.P. Singh, J.C. McClure, S. Velumani, N.R. Mathews, P.J. Sebastian // Solar Energy Materials \& Solar Cells 76, p. 293(2003).

3. X. Mathew // J. Phys. D: Appl. Phys. 33, p. 1565 (2000).

4. X. Mathew, P.J. Sebastian, A. Sanchez, J. Campos // Solar Energy Materials \& Solar Cells 59, p. 99 (1999).

5. M. Lavagna, J.P. Pique, Y. Marfaing // Solid State Electronics 20, 235 (1977).

6. W.W. Gartner // Phys. Rev. 116, p. 84 (1959).

7. D.M. Hofmann, W. Stadler, P. Chrismann, B.K. Meyer // Nucl. Instr. and Meth. A 380, p. 117 (1996).

8. M. Zha, E. Gombia, F. Bissoli, A. Zappettini, A. Zanotti // Phys. status solidi (b) 229, p. 15(2002).

9. S.M. Sze, Physics of Semiconductor Devices. Wiley, New York, 1981, p. 251, 800-803.

10. T. Toshifumi, S. Adachi, H. Nakanishi, K. Ohtsuka // Jpn J. Appl. Phys. 32, p. 3496 (1993). 